\title{
Remote Postischemic Conditioning Promotes Stroke Recovery by Shifting Circulating Monocytes to CCR2 ${ }^{+}$ Proinflammatory Subset
}

\author{
Jiwon Yang, ${ }^{1}$ Mustafa Balkaya, ${ }^{1}$ Cesar Beltran, ${ }^{1}$ Ji Hoe Heo, ${ }^{2}$ and Sunghee Cho ${ }^{1,3}$ \\ ${ }^{1}$ Burke Neurological Institute, White Plains, New York 10605, ${ }^{2}$ Department of Neurology, Yonsei University College of Medicine, Seoul 03722, Republic of \\ Korea, and ${ }^{3}$ Feil Family Brain and Mind Research Institute, Weill Cornell Medicine, New York, New York 10065
}

Brain injury from stroke is typically considered an event exclusive to the CNS, but injury progression and repair processes are profoundly influenced by peripheral immunity. Stroke stimulates an acute inflammatory response that results in a massive infiltration of peripheral immune cells into the ischemic area. While these cells contribute to the development of brain injury, their recruitment has been considered as a key step for tissue repair. The paradoxical role of inflammatory monocytes in stroke raises the possibility that the manipulation of peripheral immune cells before infiltration into the brain could influence stroke outcome. One such manipulation is remote ischemic limb conditioning (RLC), which triggers an endogenous tolerance mechanism. We observed that mice subjected to poststroke RLC shifted circulating monocytes to a CCR $2{ }^{+}$proinflammatory monocyte subset and had reduced acute brain injury, swelling, and improved motor/gait function in chronic stroke. The RLC benefits were observed regardless of injury severity, with a greater shift to a CCR2 ${ }^{+}$subset in severe stroke. Adoptive transfer of CCR2-deficient monocytes abolished RLC-mediated protection. The study demonstrates the importance of RLC-induced shift of monocytes to a CCR2 ${ }^{+}$proinflammatory subset in attenuating acute injury and promoting functional recovery in chronic stroke. The defined immune-mediated mechanism underlying RLC benefits allows for an evidence-based framework for the development of immune-based therapeutic strategies for stroke patients.

Key words: CCR2; inflammation; ischemic stroke; monocyte; recovery; remote ischemic limb conditioning

Significance Statement

Stroke is the leading cause of physical disability worldwide but has few treatment options for patients. Because remote ischemic limb conditioning (RLC) elicits endogenous tolerance in neither an organ- nor a tissue-specific manner, the immune system has been considered a mediator for an RLC-related benefit. Application of RLC after stroke increased a proinflammatory CCR2 ${ }^{+}$ monocyte subset in the blood and the brain. RLC reduced acute stroke injury and promoted motor/gait function during the recovery phase. The RLC benefits were absent in mice that received CCR2-deficient monocytes. This preclinical study shows the importance of $\mathrm{CCR} 2^{+}$proinflammatory monocytes in RLC benefits in stroke and provides a therapeutic RLC platform as a novel immune strategy to improve outcomes in stroke patients.

\section{Introduction}

Ischemic conditioning is an adaptive endogenous protection, in which sublethal ischemia provides protection to organs or tissues

\footnotetext{
Received 0ct. 18, 2018; revised June 17, 2019; accepted July 26, 2019.

Author contributions: J.Y. and S.C. designed research; J.Y., M.B., and C.B. performed research; J.Y. analyzed data; J.Y. wrote the first draft of the paper; J.Y., M.B., J.H.H., and S.C. edited the paper; J.Y. and S.C. wrote the paper

This work was supported by National Institutes of Health Grants HL082511, NS095359, and NS077897 to S.C., National Research Foundation of Korea funded by the Ministry of Education Basic Science Research Program 2013R1A6A3A03059436 to J.Y., and American Heart Association Postdoctoral Fellowship 15P0ST25680020 to J.Y. We thank Dr. John Cave for comments and editorial assistance; and Dr. Yang Guo for technical assistance.

The authors declare no competing financial interests.

Correspondence should be addressed to Sunghee Cho at suc2002@med.cornell.edu.

J. Yang's present address: The Jackson Laboratory, 1650 Santa Ana Avenue, Sacramento, CA 95838.
}

from subsequent full insults (Dirnagl et al., 2009). The conditioning effect on ischemia-reperfusion injury also occurs remotely, and preclinical and clinical studies show that it protects multiple organs, including the brain, heart, and kidney (Zhou et al., 2011; Hoda et al., 2014; England et al., 2017; Oliveira et al., 2017). Ischemic conditioning applied before (pre), during (per), or after (post) insults elicits tolerance across these paradigms. Significant clinical attention has been given to remote ischemic limb conditioning (RLC) due to its noninvasive nature and potential therapeutic applications in stroke patients. 
Several preclinical studies have shown that ischemic conditioning reduces infarct size (Hoda et al., 2012; Sun et al., 2012). Understanding of the mechanism(s) conferring RLC-induced benefits is currently limited, but the observation of cross-organ protection from a distant conditioning site suggests the involvement of neural and humoral factors. In cardiac ischemia and stroke studies, increased blood flow was associated with RLCmediated infarct reduction (Hoda et al., 2012; Kono et al., 2014). Circulation factors, such as nitrite and bradykinin, are also implicated in the protective effects (Starkopf et al., 1997; Rassaf et al., 2014; Hess et al., 2016). RLC induces a reduction in macrophage and neutrophil infiltration of the heart, as well as a reduction of both circulating $\mathrm{CD} 3{ }^{+} / \mathrm{CD}^{+} \mathrm{T}$ cells and NK cells following stroke (Wei et al., 2011; Liu et al., 2016). Ischemic conditioning also dramatically increases stroke-induced B cells and noninflammatory resident monocytes in the blood (Liu et al., 2016). Together, these studies suggest that immune cell modulation underlies RLC-mediated protection in ischemic injury.

The role of immune cells in cerebral ischemia has been equivocal. Enhanced inflammation is associated with exacerbated ischemic outcome (McColl et al., 2007), but postischemic inflammation is also considered to be a necessary process for tissue remodeling (Zhao et al., 2006; Ekdahl et al., 2009). In myocardial infarction, RLC-induced protective effects are associated with an attenuated inflammatory response by decreasing the number of infiltrated macrophages and neutrophils to the infarct (Wei et al., 2011). In contrast, conditioning-induced endogenous neuroprotection is closely linked to inflammation in stroke (Ohtsuki et al., 1996; Bordet et al., 2000). Inflammatory mediators, such as cytokines, chemokines, and toll-like receptors, are critical for conditioninginduced protection, and the absence of these mediators abolishes the conditioning effect (Wang et al., 2015; McDonough and Weinstein, 2016). Thus, these studies support a close link between proinflammation and conditioning-mediated protection.

Monocytes are a major source of inflammatory mediators and are key players in stroke pathology. Circulating monocytes in mice are found in two distinct subsets: Classical monocytes express a high level of Ly-6C and also express C-C chemokine receptor Type $2\left(\mathrm{CCR}^{+}{ }^{+}\right)$. The classical monocytes (Ly-6C ${ }^{\text {high} /}$ $\mathrm{CCR} 2^{+}$) are recruited into the inflamed tissue in a CCR2dependent manner and become classically activated M1 macrophages. This subset produces inflammatory and cytotoxic factors and contributes to inflammation. Nonclassical monocytes express a low level of Ly-6C and do not express CCR2. This antiinflammatory subset patrols blood vessel lumens for immune surveillance, maintains vascular homeostasis, and facilitates tissue repair and remodeling (Gordon, 2007; Auffray et al., 2009). Stroke increases the number of circulating monocytes, which infiltrate the brain. Peak infiltration occurs a few days after stroke (Urra et al., 2009; Kim et al., 2014). Literature indicates that Ly-6C ${ }^{\text {high }}$ subset limits ischemic damage by assisting microvasculature stability and debris removal (ElAli and Jean LeBlanc, 2016). Similarly, mice with depleted peripheral Ly-6C high/ $\mathrm{CCR}^{+}$monocytes have worse stroke recovery (Chu et al., 2015; Wattananit et al., 2016), supporting the potential importance of CCR $2^{+}$monocytes in functional benefits. Monocyte trafficking to the injured brain can potentially lead to immune depression and infection in the periphery (Aslanyan et al., 2004; Chamorro et al., 2006). Thus, these findings indicate the importance of monocyte subset distribution between the brain and periphery for eliciting necessary inflammation in the brain and for counteracting peripheral infection. This balance is disrupted in stroke. The present study therefore investigated the effect of postisch- emic RLC on monocyte changes in the periphery and the brain after stroke, and how the changes modify acute and long-term stroke outcome. Here, we report that an acute shift of circulating monocytes toward CCR ${ }^{+}$proinflammatory subset mediates RLC-induced functional benefits in stroke.

\section{Materials and Methods}

Experimental design. Initial experiments with C57BL/6 mice were done in males, and further mechanistic experiments were done in both males and females. A total of 283 mice were included in the study: 36 mice ( 8 sham-conditioning [Sham] and 28 RLC) to study the effect of RLC without stroke, 101 mice (50 Sham and 51 RLC) to study monocyte subset changes and/or injury size measurement in vivo, 12 mice (5 Sham and 7 RLC) to study in vitro monocytes conversion, 39 mice (20 Sham and 19 RLC) for long-term behavioral tests, 37 mice (18 Sham and 19 RLC) for injury size-dependent monocyte subset change, 18 mice (4 WT Sham, 4 WT RLC, 5 CCR 2 KO Sham, and 5 CCR2 KO RLC) for WT and CCR2 KO adoptive transfer study without stroke, 16 mice ( 8 Sham [ 3 male and 4 female] and 8 RLC [ 4 male and 4 female]) to study monocyte subset changes in CCR2 KO adoptive transfer mice, and 24 mice (11 Sham [6 male and 5 female] and 13 RLC [ 9 male and 4 female]) for the CCR2 KO adoptive transfer long-tern behavioral study.

Animals. A priori calculations for sample sizes indicated that a minimum of 10 animals per group was needed for injury size measurement and behavioral testing to reach power 0.80 at a significance level of $<0.05$, based on a $27 \%$ difference in mean and a $20 \%$ SD at the $95 \%$ confidence level. The use of animals and procedures was approved by the Institutional Animal Care and Use Committee of Weill Cornell Medicine and in accordance with the Institutional Animal Care and Use Committee, National Institutes of Health, and Animal Research: Reporting of in vivo experiment guidelines. Experiments were performed in 11- to 13week-old male and female C57BL/6 mice (The Jackson Laboratory) and CCR2 KO mice (The Jackson Laboratory). Mice were housed at the institute's animal facility, which monitors and maintains temperature, humidity, and $12 \mathrm{~h}$ light/dark cycles. A maximum of 5 mice were housed per cage, and all cages contained an individual ventilating system and irradiated bedding (1/800 Bed o'Cobs, The Anderson). Sterilized food (PicoLab Rodent diet 5053, LabDiet) and water were freely accessible in their cage. Animals were randomly selected for different experimental groups. Animals' identity and treatment were blinded to the person who cryosectioned brains and assessed stroke outcomes.

Transient middle cerebral artery occlusion (MCAO). Mice were subjected to transient MCAO under anesthesia using isoflurane, as previously described (Kim et al., 2014; Woo et al., 2016). Briefly, a fiber optic probe was glued to the parietal bone $(2 \mathrm{~mm}$ posterior and $5 \mathrm{~mm}$ lateral to the bregma) and connected to a Laser-Doppler Flowmeter (Periflux System 5010; Perimed) for continuous monitoring of cerebral blood flow in the ischemic territory. For MCAO, a 6-0 Teflon-coated black monofilament surgical suture (Doccol) was inserted into the exposed external carotid artery. The monofilament was advanced into the internal carotid artery, where it was wedged into the cerebral arterial circle to obstruct the origin of the middle cerebral artery for $30 \mathrm{~min}$. Following occlusion, the filament was withdrawn to allow reperfusion. Buprenorphine $(0.5 \mathrm{mg} /$ $\mathrm{kg}$, s.c., every $12 \mathrm{~h}$ for first $48 \mathrm{~h}$ after surgery) and bupivacaine $(0.1 \mathrm{ml}$ of $0.25 \%-0.5 \%$, s.c., on incision site before incision) were administered as preoperation and postoperation analgesics. Mice were then placed in a recovery cage until they regained consciousness and resumed activity. Using a rectal probe controlled by a Masterflex pump and thermistor temperature controller (Cole-Parmer), body temperature was maintained at $36.5 \pm 0.5^{\circ} \mathrm{C}$ during $\mathrm{MCAO}$ and recovery after the surgery. The mice were then returned to their home cages, where they were previously housed together. To hydrate animals after surgery, hydrogel $\left(\mathrm{ClearH}_{2} \mathrm{O}\right)$ was provided with moistened food, and saline was injected subcutaneously when mice were severely dehydrated. Only animals that exhibited $>80 \%$ reduction in cerebral blood flow during MCAO and $>80 \%$ reperfusion $10 \mathrm{~min}$ after reperfusion were included in the study. Body weight was measured at the time of MCAO and daily up to $7 \mathrm{~d}$, weekly up to 4 weeks, and then monthly up to 4 months after stroke. 
A
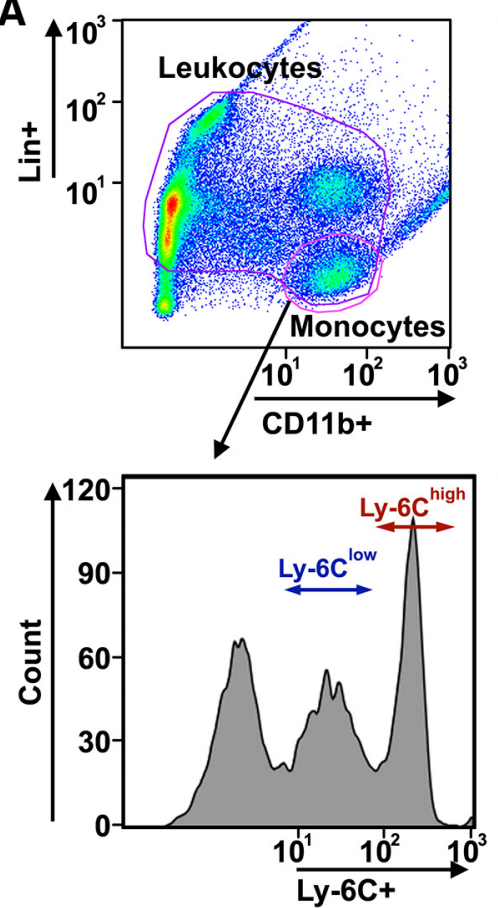

B

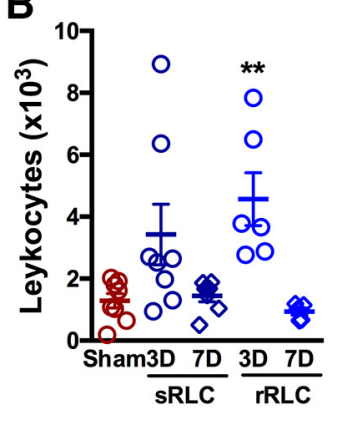

C

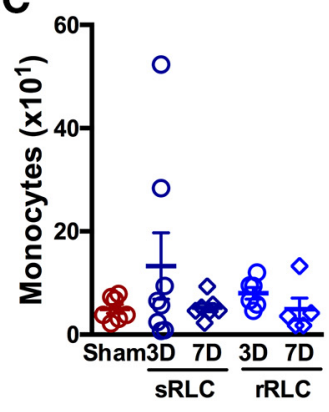

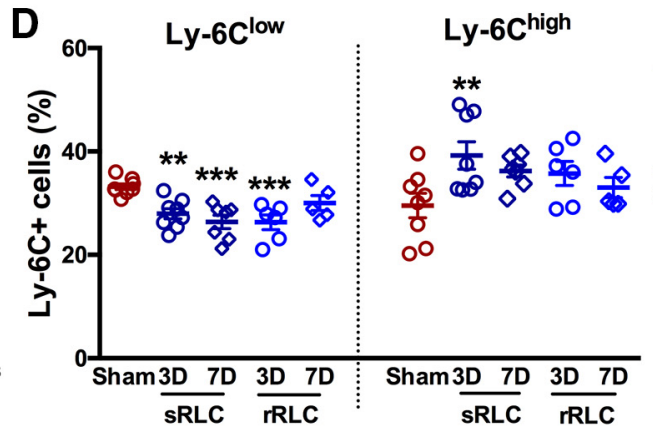

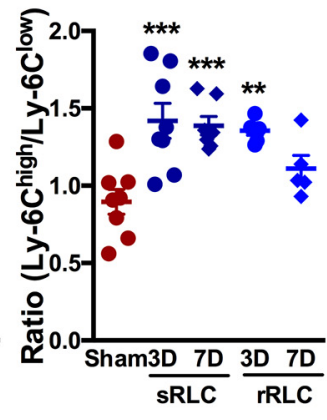

Figure 1. RLC induces a shift of circulating monocytes toward a proinflammatory subset in nonstroked animals. $A$, Gating strategy for leukocytes (Lin ${ }^{+}$and CD11b ${ }^{+}$population) and monocytes (Lin ${ }^{-} /$CD11b ${ }^{+}$cells). Monocyte subsets in the blood were further analyzed for Ly-6C expression. Single RLC (sRLC) or daily repeated RLC (rRLC) was applied, and immune cells were analyzed at 3 or $7 \mathrm{~d}$ in the blood. $\boldsymbol{B}, \boldsymbol{C}$, Number of leukocytes $(\boldsymbol{B})$ and monocytes $(\boldsymbol{C})$ in the blood per microliter. $\boldsymbol{D}$, Monocyte subsets and ratios of $L y-6 C^{\text {high }} / L y-6 C^{\text {low }}$ in the blood (Figure $1-1$, available at https://doi.org/10.1523/JNEUROSCI.2699-18.2019.f1-1). Number of leukocytes ( $\boldsymbol{A})$ and monocytes ( $\boldsymbol{B})$ in the spleen per microliter. Monocyte subsets and ratios of Ly- $6 C^{\text {high }} / L y-6 C$ low in the spleen. One-way ANOVA: ${ }^{* *} p 0.01$ versus Sham; ${ }^{* *} p<0.001$ versus Sham.

RLC. The RLC protocol used in this study was adapted from clinical trials in stroke or myocardial infarct patients (Clinical trial ID NCT01071265, NCT01654666, NCT00435266, NCT00997217, NCT01321749, etc.). RLC was performed on the left hindlimb of the isoflurane-anesthetized mice by applying total 5 cycles of inflation and deflation $(200 \mathrm{mmHg}, 5 \mathrm{~min} \times 5 \mathrm{~min}$ interval between cycles) using a small blood pressure cuff (Hokanson). RLC was applied either single or repeatedly by a daily application. For a poststroke application, RLC was applied $2 \mathrm{~h}$ after reperfusion. The Sham group exposed to the same duration of isoflurane served as a control.

Splenectomy and adoptive transfer of CCR2 KO splenocytes into $C 57 B L / 6$ mice. Splenectomy and adoptive transfer of splenocytes were performed as previously reported (Kim et al., 2014). Briefly, 13-week-old mice were anesthetized with isoflurane and an $\sim 1 \mathrm{~cm}$ incision was made on the left side of the abdominal cavity under the rib cage. The spleen was removed, and the splenic vessels were cauterized. As an analgesic, meloxicam $(5 \mathrm{mg} / \mathrm{kg}$, p.o.) was administered right before the surgery, in addition to buprenorphine $(0.5 \mathrm{mg} / \mathrm{kg}$, s.c., every $12 \mathrm{~h}$ for first $48 \mathrm{~h}$ after surgery) and bupivacaine ( $0.1 \mathrm{ml}$ of $0.25-0.5 \%$, s.c., on incision site before incision). Splenocytes collected from a CCR2 KO mouse were immediately transferred via retroorbital injection to an asplenic C57BL/6 mouse. The mice were then subjected to MCAO, followed by Sham or RLC.

Brain acute injury size measurement. Three days after MCAO, brains spanning $\sim 7.2 \mathrm{~mm}$ rostrocaudal $(\sim 3.1 \mathrm{~mm}$ and extending to $-4.2 \mathrm{~mm}$ from bregma) were cryosectioned at $20 \mu \mathrm{m}$ thickness and were collected serially at $600 \mu \mathrm{m}$ intervals. A total of 13 brain sections were used for infarct size and swelling measurements, which were performed with Axiovision software (Carl Zeiss) as previously described (Kim et al., 2014, 2015). Collected brain sections were examined with a phasecontrast microscopy to visualize infarcted area. Infarct territory was traced in each section to calculate the infarct area. Infarct volume was determined by multiplying the infarct area $\left(\mathrm{mm}^{2}\right)$ in each section by the distance $(0.6 \mathrm{~mm})$ to the next section and adding the results from all the sections. Infarct volume was corrected for edema by subtracting the hemispheric difference. To calculate percent hemispheric swelling, the difference between ipsilateral and contralateral hemispheric volume was divided by contralateral hemisphere volume and multiplied by 100 .

Flow cytometry. Flow cytometry analysis for monocytes in the spleen and blood was performed according to the methods previously described (Kim et al., 2014). Spleen and blood cells were incubated with 10\% FBS in PBS with mouse BD Fc Block (rat anti-mouse CD16/CD32, 553142) at $4^{\circ} \mathrm{C}$ for $30 \mathrm{~min}$. Single cells were subsequently incubated with: (1) a mixture of phycoerythrin-conjugated antibodies (PE-Lin) against T cells (PE rat anti-mouse CD90.2, 553005), B cells (PE rat anti-mouse CD45R/ B220, 553089), natural killer cells (PE mouse anti-mouse NK-1.1, 557391, and PE rat anti-mouse CD49b, 553858), and granulocytes (PE rat anti-mouse Ly-6G, 551461); (2) phycoerythrin and cyanine 7 -conjugated CD11b (PE-Cy7 rat anti-mouse CD11b, 552850 or mouse CD11b-PE-Vio770, 130-109-287, Miltenyi Biotec); and (3) FITCconjugate Ly-6C (FITC rat anti-mouse Ly-6C, 553104) for $40 \mathrm{~min}$. The monocyte population was gated as Lin-negative/CD11b-positive ( $\mathrm{Lin}^{-}$/ $\mathrm{CD}_{1} 1 \mathrm{~b}^{+}$), and the selected population was further analyzed for the distribution of Ly-6C high and Ly-6C low monocyte subsets using FITCLy-6C antibody. For the brain tissue, single brain cells from contralateral and ipsilateral hemispheres were prepared using Neural Tissue Dissociation Kit (P) (Miltenyi Biotec) with gentleMACS Octo Dissociator (Miltenyi Biotec) according to the manufacturer's instructions. To obtain mononuclear cells from brain single-cell suspension, discontinuous Percoll gradient method (30\%, 37\%, and 70\%; GE Healthcare) was used (Woo et al., 2016). To detect myeloid lineage cells in the CNS, PEconjugated CD45 (PE rat anti-mouse CD45, 553081) and PE-Cy7CD11b or CD11b-PE-Vio770 antibodies were used to identify CD11b ${ }^{+}$ and CD45 low expressed population $\left(\mathrm{CD} 45^{\text {low }}\right)$ and $\mathrm{CD} 11 \mathrm{~b}^{+}$and CD45 high expressed population (CD45 high $)$, which were further analyzed with the FITC-Ly-6C antibody. Only live cells were included using Live/Dead Fixable dead cell stain kit (Thermo Fisher Scientific). A total of 100,000 cells were counted per each sample using a flow cytometer (Accuri C6, BD Biosciences or MACSQuant VYB, Miltenyi Biotec). All antibodies used for flow cytometry were purchased from BD Biosciences unless 
A

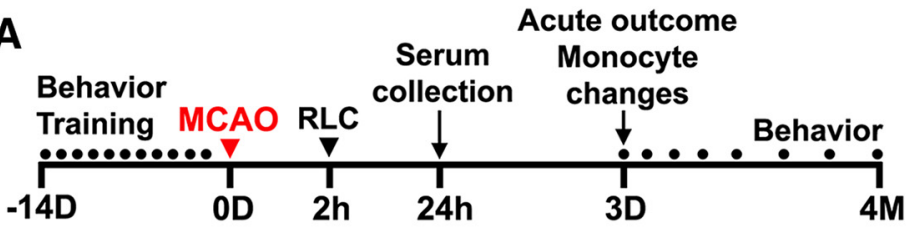

B

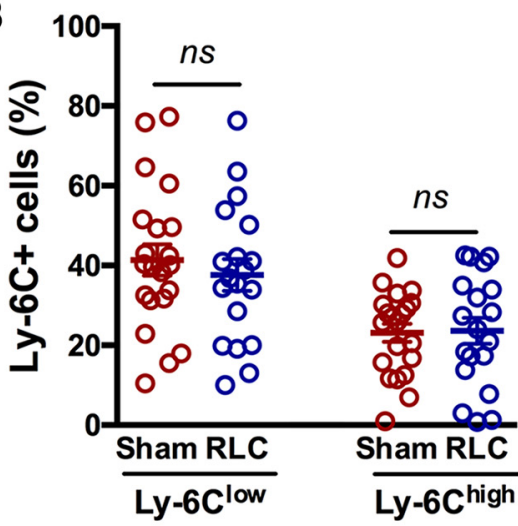

C

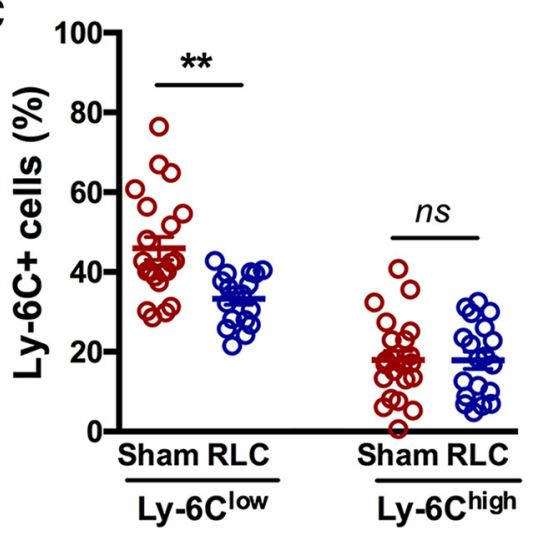

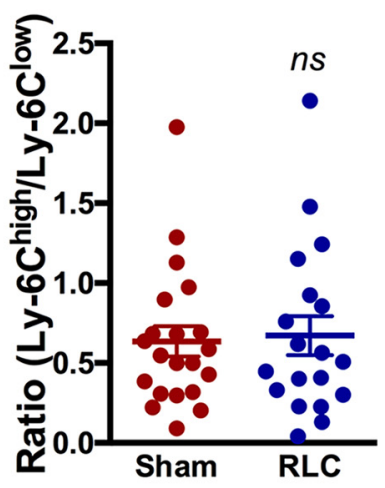

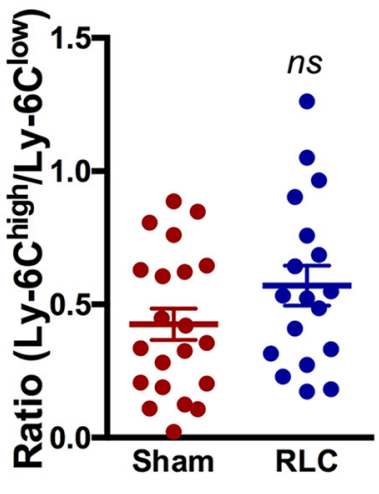

Figure 2. RLC-induced peripheral monocyte subset changes in stroked mice. $\boldsymbol{A}$, Experimental timeline. $\boldsymbol{B}, \boldsymbol{C}$, Monocyte subset analysis in the spleen $(\boldsymbol{B})$ and the blood $(\boldsymbol{C})$ of Sham and RLC mice. ${ }^{* *} p<0.01$ ( $t$ test). ns, not significant.

noted otherwise. For multicolor compensation, Anti-Rat and AntiHamster Ig $\kappa /$ Negative Control Compensation Particles Set (552845, BD Biosciences), Anti-Mouse Ig $\kappa /$ Negative Control Compensation Particles Set (552843, BD Biosciences), and MACS Comp Bead Kit (130-107-755, Miltenyi Biotec) were used. Flow cytometry data were analyzed using FlowJo software (FlowJo).

Assessment of monocyte subsets in vitro. Sera were collected from Sham or RLC mice $24 \mathrm{~h}$ after MCAO surgery and were stored at $-80^{\circ} \mathrm{C}$ until use. Naive splenocytes were collected and prepared for single-cell suspensions, as described above. Following single splenocyte collection, $\mathrm{CD}_{11 \mathrm{~b}}{ }^{+}$splenocytes were isolated using CD11b MicroBeads (130-049601, Miltenyi Biotec) according to the manufacturer's instructions. $\mathrm{CD}_{11} \mathrm{~b}^{+}$splenocytes were then incubated without serum, with Sham serum, or with RLC serum for $4 \mathrm{~h}$ at $37^{\circ} \mathrm{C}$ with $5 \% \mathrm{CO}_{2}$. After $4 \mathrm{~h}$ of incubation, cells were collected and stained for flow cytometry as described above. A total of 20,000 cells were analyzed by a flow cytometer. Sera from each group ( $n=3$ or 4 ) were combined together, and assays were quadruplicated. Two independent experiments were performed.

Assessment of $M C P-1$ and $I L-1 \beta m R N A$ levels. Total RNA from brain tissues ( $3 \mathrm{~d}$ after stroke) was reverse-transcribed using QuantiTech reverse transcription kits (QIAGEN), and relative mRNA levels were measured using fluorescent TaqMan real-time RT-PCR (Woo et al., 2016). TaqMan predeveloped assay reagents, probes, and gene-specific primers for MCP-1 (Mm00441242_m1) and IL-1 $\beta$ (Mm00434228_m1) were used (Invitrogen). $\beta$-Actin was used as an internal control for sample normaliza tion using the following formula: Value $=$

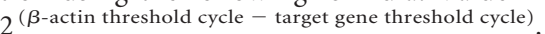

PCR was performed in an Applied Biosystems 7500 fast real-time PCR system (Invitrogen) using FastStart Universal Probe Master Mix (Roche Applied Science).

Behavior assessment. RotaRod performance and gait analyses were conducted as previously described (Qin et al., 2014; Kim et al., 2015). The RotaRod device (ENV-576M, Med Associates) accelerated from 4 to $40 \mathrm{rpm}$ over the course of $5 \mathrm{~min}$. The animals were pretrained for $7 \mathrm{~d}$ before MCAO, and performances for each animal on the last $3 \mathrm{~d}$ before ischemia were recorded and averaged for the preischemic baseline for that animal. The latency to fall from the rod was averaged from five trials. Gait changes were assessed using a Catwalk XT gait analysis system (Noldus Information Technology). The Catwalk measures the kinematics of gait while the mouse walks from one end of a flat surface to another. Footprints were identified and digitally recorded when the light changes with paw placement on the glass-walking surface. Regularity index was used to gauge overall gait function. For detecting asymmetry, paw contact area, intensity of paw pressure, stride length, and swing speed of the affected side of the body (left side) were used. Mice were pretrained for 2 weeks to cross an illuminated glass walkway three consecutive times, which is a necessary step to obtain quantifiable digital signals. The average recording on the last $3 \mathrm{~d}$ before stroke was used for a preischemic baseline for each animal. Poststroke testing was performed during acute (days), subacute (weeks), and recovery (months) phases following the stroke. Behavioral data were presented as a percentage of preischemic baseline (\% pre) to account for interanimal variability.

Statistics. Comparisons between two groups were statistically evaluated using Student's $t$ test. Multiple comparisons were examined using ANOVA followed by post hoc Bonferroni's multiple comparisons. Two-way ANOVA with post hoc Bonferroni's comparison was performed for (1) effect of stroke and (2) effect of RLC. Statistical analyses were performed using Prism software (GraphPad Software). Infarct volume and percent hemispheric swelling were reported as mean $\pm 95 \% \mathrm{CI}$, and all other data were reported as mean \pm SEM. Differences were considered significant if $p<0.05$.

\section{Results}

RLC shifts circulating monocytes toward proinflammatory subset in nonstroked animals

We first investigated the effect of single or repeated RLC on peripheral immune cell changes in nonstroked animals. Using a gating strategy shown in Figure $1 A$, we identified the leukocyte (Lin ${ }^{+}$and $\mathrm{Lin}^{-} / \mathrm{CD}_{11 \mathrm{~b}^{+}}$: T cells, B cells, neutrophils, NK cells, and monocytes) and monocyte ( $\mathrm{Lin}^{-} / \mathrm{CD} 11 \mathrm{~b}^{+}$) populations in the spleen. RLC did not change either leukocyte or monocyte counts at 3 or $7 \mathrm{~d}$ after initial RLC (Fig. 1-1A, available at https:// doi.org/10.1523/JNEUROSCI.2699-18.2019.f1-1; Fig. 1-1B, available at https://doi.org/10.1523/JNEUROSCI.2699-18.2019.f1$1)$. Analysis of monocyte subsets showed that RLC increased Ly- $6 C^{\text {high }}$ subset $3 \mathrm{~d}$ after either single or repeated RLC, but the ratio of proinflammatory to anti-inflammatory monocyte sub- 

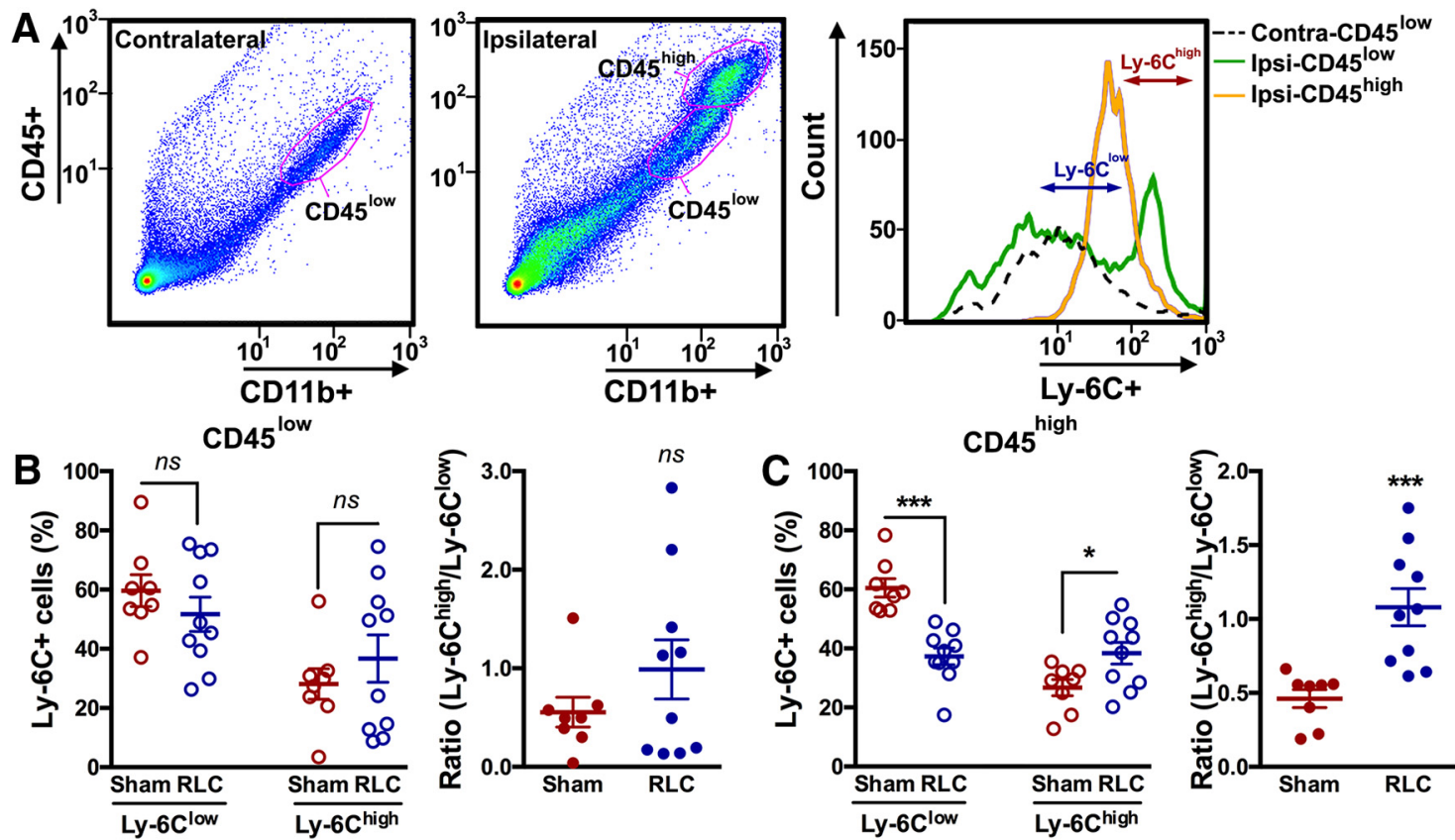

Figure 3. RLC-induced changes in infiltrated monocyte/macrophage subsets in the brain following stroke. $\boldsymbol{A}$, Gating strategies for resident microglia (CD45 ${ }^{\mathrm{low}}$ ) and infiltrated monocytes/ macrophages (CD45 high) at $3 \mathrm{~d}$ after stroke with subanalysis of Ly- 6 C expressions. B, C, Percentage of Ly- 6 C subsets and ratios of Ly- $6 C^{\text {high }}$ over Ly- $6 C^{\text {low }}$ subset in the CD45 ${ }^{\text {low }}(\boldsymbol{B})$ and CD45 high $(\boldsymbol{C})$ population in the ipsilateral hemisphere. $t$ test: ${ }^{*} p<0.05 ;{ }^{* * *} p<0.001$. Contra, Contralateral hemisphere; Ipsi, ipsilateral hemisphere. ns, not significant.

sets was unchanged (Fig. 1-1C, available at https://doi.org/ 10.1523/JNEUROSCI.2699-18.2019.f1-1). We next determined the effect of RLC in the blood. Repeated RLC, but not single RLC, significantly increased total leukocyte counts at $3 \mathrm{~d}$ without changes in monocyte counts (Fig. $1 B, C$ ). RLC reduced Ly-6C ${ }^{\text {low }}$ subset with a concomitant increase in the Ly- $6 C^{\text {high }}$ subset $3 \mathrm{~d}$ after single conditioning, which led to a significant shift in circulating monocytes toward a proinflammatory status (Fig. 1D). The shift was greater with single RLC than with repeated RLC, and the effect of single RLC lasted up to $7 \mathrm{~d}$. The results showed that single RLC induced robust and long-lasting changes in monocyte subsets in the periphery at $3 \mathrm{~d}$, and this RLC paradigm was used for the rest of the study.

\section{RLC decreases circulating Ly- $6 C^{\text {low }}$ monocytes in the stroked mice}

The effect of single RLC on monocyte subset change was determined in mice with transient focal stroke (Fig. 2A). RLC did not change proinflammatory and anti-inflammatory monocyte subsets in the spleen after stroke (Fig. $2 B$ ). RLC significantly decreased Ly-6 $\mathrm{C}^{\text {low }}$ monocytes in the blood, but there was neither an increase in Ly-6C $\mathrm{C}^{\text {high }}$ subset nor a monocyte shift toward a proinflammatory subset in the blood (Fig. 2C).

RLC reduces $L y-6 C^{\text {low }}$ and increases $L y-6 C^{\text {high }}$ subsets in the stroked brain

Stroke induces dynamic trafficking of immune cells from the periphery to the injured brain (Kim et al., 2014). The absence of an RLC-induced monocyte shift in the circulation of stroked mice suggested that there was trafficking of Ly- $6 \mathrm{C}^{\text {high }}$ monocytes to the injured brain. In the brain contralateral to the stroke lesion, $\mathrm{CD}_{11} \mathrm{~b}^{+}$cells were mostly a CD $45^{\text {low }}$ population. By contrast, cells in the ipsilateral hemisphere were CD $45^{\text {low }}$ and CD $45^{\text {high }}$, and likely represented the resident microglia and infiltrated macrophages (Fig. 3A) (Kim et al., 2014). Unlike the periphery, there were no distinct Ly-6C $\mathrm{C}^{\text {low }}$ and Ly-6C $\mathrm{C}^{\text {high }}$ subsets in the brain (Fig.
$3 A$ ), which reflected the downregulation of Ly-6C expression in the brain as monocytes differentiate to tissue macrophages (Gliem et al., 2016). By using the Ly-6C gating strategy defined by periphery in Figure $1 A$, we found that RLC did not affect Ly-6C subsets in the CD45 ${ }^{\text {low }}$ population in the contralateral hemisphere (Sham vs RLC, \%Ly-6C ${ }^{\text {low }}, 61.21 \pm 3.12 \%$ vs $64.23 \pm$ $1.73 \%, p=0.3859 ; \%$ Ly- $6 C^{\text {high }} 14.73 \pm 2.98 \%$ vs $14.27 \pm 1.25 \%$, $p=0.8782)$. In the ipsilateral hemisphere, RLC did not affect Ly-6C ${ }^{\text {low }}$ and Ly- $6 C^{\text {high }}$ subsets in the CD $45^{\text {low }}$ resident microglia population or the ratio between the subsets (Fig. $3 B$ ). In the CD $45^{\text {high }}$ population, RLC decreased the Ly- $6 C^{\text {low }}$ and increased Ly-6C ${ }^{\text {high }}$ subsets, which showed a monocyte shift to proinflammatory status (Fig. 3C). The RLC-induced proinflammatory shift only in the infiltrating CD $45^{\text {high }}$ population suggests a shift in the blood before their trafficking to the brain.

\section{RLC converts anti-inflammatory monocytes to a proinflammatory subset in vitro}

We further confirmed the in vivo findings that RLC induces a shift in monocyte subsets within the periphery after stroke by using in vitro splenocyte culture, in which there is no monocyte trafficking (Fig. 4A). Exposure of the sera obtained from stroke/ $\mathrm{RLC}$ mice to $\mathrm{CD} 11 \mathrm{~b}^{+}$splenocytes showed a reciprocal reduction of Ly-6C ${ }^{\text {low }}$ and an increase of Ly-6C ${ }^{\text {high }}$ monocytes compared with the cells exposed to stroke/Sham sera (Fig. 4B). These changes of monocyte subsets by treating with stroke/RLC serum led to a significant shift of monocytes to the proinflammatory subset.

\section{RLC reduces brain injury and improves functional recovery}

Effect of RLC on acute stroke outcomes was determined $3 \mathrm{~d}$ after stroke. Compared with Sham mice, animals with RLC showed a moderate, but significant, reduction in infarct size and swelling (Fig. 5). The improved acute stroke outcome was associated with reduced $\mathrm{MCP}-1$ and IL- $1 \beta$ gene expression, proinflammatory mediators, in the ipsilateral hemisphere at this time (Sham vs 

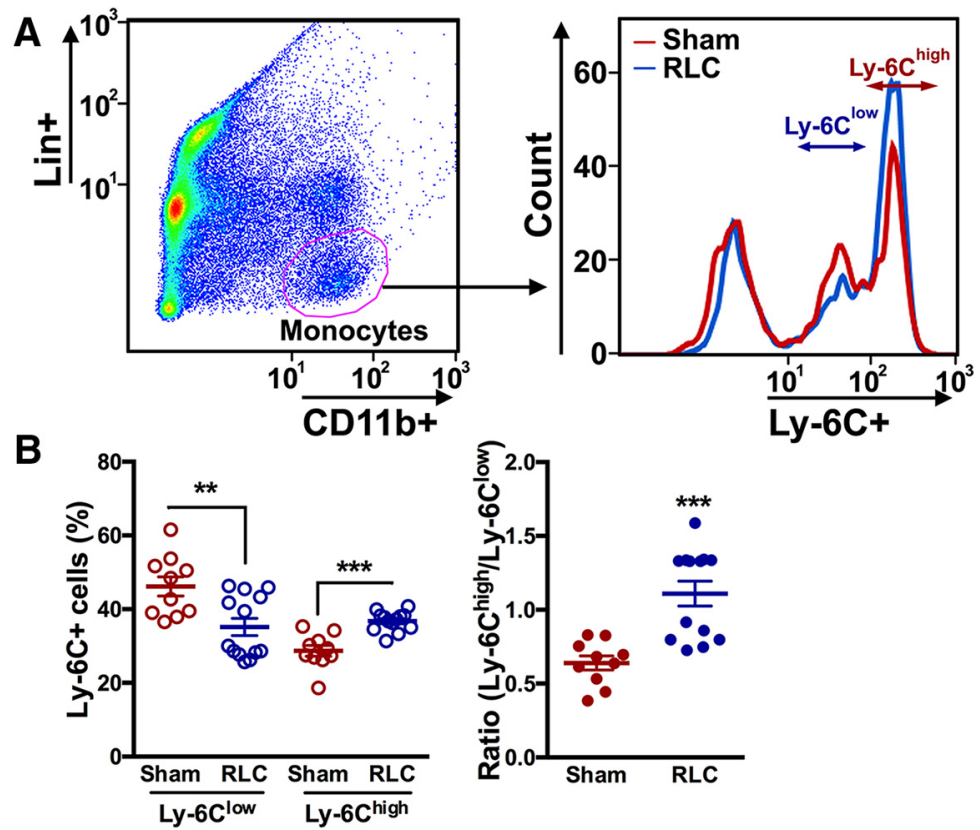

Figure 4. RLC converts anti-inflammatory monocytes to a proinflammatory subset in vitro. $A, A$ gating strategy to identify monocyte subsets in the CD11b ${ }^{+}$splenocyte culture. $B$, Percentage of Ly- $6 C$ subsets and the ratios of Ly- $6 C^{\text {high }}$ over Ly- $6 C^{\text {low }}$ subset. Sera from sham or RLC mice were collected and treated to the naive splenocytes. $t$ test: ${ }^{* *} p<0.01 ;{ }^{* * *} p<0.001$.

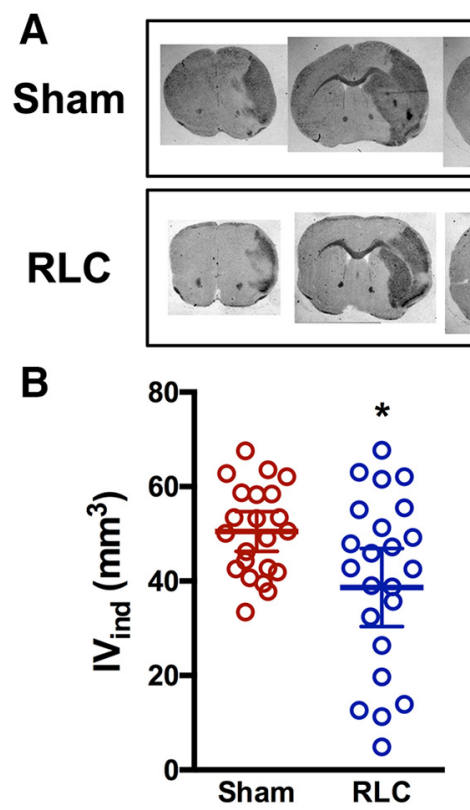

Figure 5. Effect of RLC on acute stroke outcomes. $A$, Representative section of every other brain section collected at $3 \mathrm{~d}$ after stroke in Sham and RLC mice. $\boldsymbol{B}, \boldsymbol{C}$, Infarct volume $(\boldsymbol{B})$ and percent hemispheric swelling $(\boldsymbol{C})$ measured from 13 serial sections in Sham and RLC mice. Data are mean \pm 95\% Cl (Figure 5-1, available at https://doi.org/10.1523/JNEUROSCI.2699-18.2019.f5-1). ${ }^{*} p<0.05$ (t test). Stroke-induced body weight changes. Body weights are presented as percentage of preischemic baseline of each animal. IV ind Indirect infarct volume.

RLC: MCP- $1 \times 10^{-3}, 1.51 \pm 0.71$ vs $\left.0.7 \pm 0.53, p<0.05, n=7\right)$ : IL- $1 \beta \times 10^{-4}, 1.36 \pm 0.97$ vs $0.31 \pm 0.21 p<0.001, n=6$ ). Stroke-induced acute body weight loss and body weight gain during a recovery period were similar between the Sham and RLC groups (Fig. 5-1, available at https://doi.org/10.1523/ JNEUROSCI.2699-18.2019.f5-1). Motor function assessment by RotaRod performance test showed that both groups had similar motor deficit during acute and subacute periods through 2 weeks, after which RLC mice showed significantly enhanced motor function that lasted up to 4 months after stroke (Fig. $6 A)$. RLC application also improved interlimb coordination, as determined by the regularity index (Fig. 6B). Further analyses of gaits of forelimb and hindlimb of the impaired side (left side of the body due to right MCAO) showed fewer acute deficits and a significant improvement of gaits in the maximum contact area, mean intensity, stride length, and swing speed during the recovery period (Fig. 6C). Together, the behavior assessments demonstrate that a single poststroke RLC application produced long-lasting functional improvement in chronic stroke.

\section{RLC enhances functional recovery across injury severity}

To identify potential adverse events of RLC application in severe stroke injury, we compared behavior outcome in RLC animals with moderate and severe brain injury. Both the literature and our preclinical data showed that strokeinduced acute body weight loss is positively correlated with infarct size (Jönsson et al., 2008; Qin et al., 2014). In our analysis of C57BL/6 male mice, there was a significant correlation of body weight loss at $3 \mathrm{~d}$ after stroke with infarct volume $\left(p<0.0001, R^{2}=\right.$ $0.1598)$ and brain swelling $(p<0.0001$, $R^{2}=0.3608$ ) (Yang et al., 2019). Based on these observations, we retrospectively divided stroke/RLC mice into two groups according to the extent of body weight loss at $3 \mathrm{~d}$ after stroke: $<15 \%$ of body weight loss for moderate injury ( $n=7$ ), and $\geq 15 \%$ of body weight loss for severe injury group $(n=13)$. Subanalysis of RotaRod performance showed that motor function improvement was different between RLC with moderate injury and RLC with severe injury for up to 3 weeks after stroke. After this time point, however, no significant difference was observed from 1 to 4 months (Fig. 7A). Additionally, mice with severe body weight loss showed worse interlimb coordination, but this recovered to baseline during a recovery phase (Fig. $7 B)$. Gait parameters of the individual limb on the affected side of the body also showed greater deficits in the severe weight loss group compared with lower weight loss animals in the early time points following the stroke, and then showed profound recovery at later recovery stages (Fig. $7 C$ ). The functional enhancements in the chronic recovery stage with mice having either moderate or severe weight loss indicate that RLC is a feasible therapy that can promote functional recovery across injury severities. 


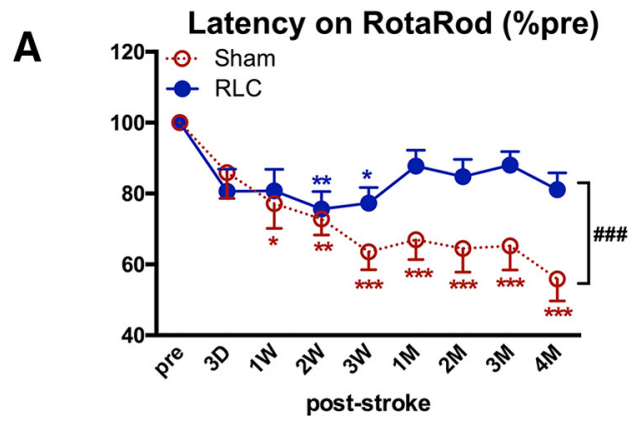

C Max Contact Area (\%)
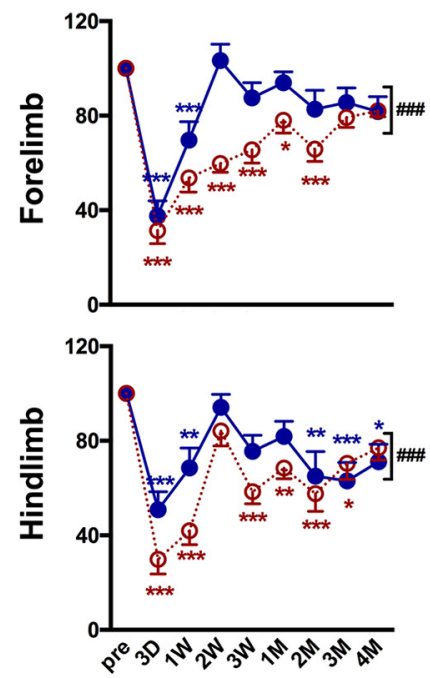

Mean Intensity (\%)
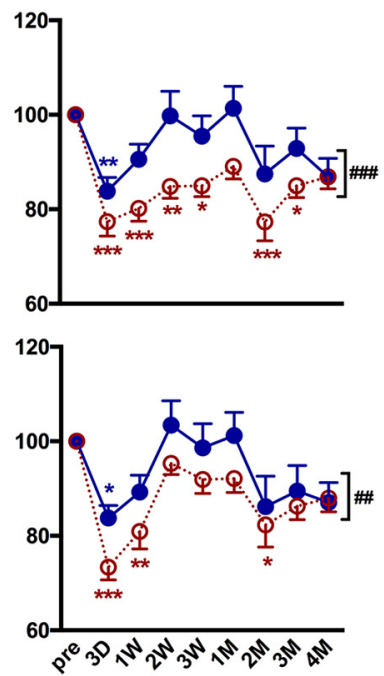

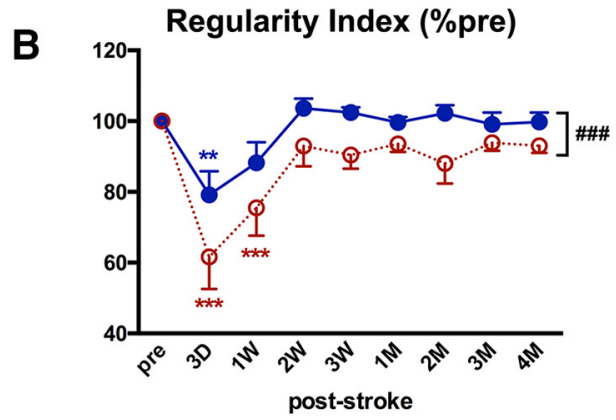

Stride Length (\%)
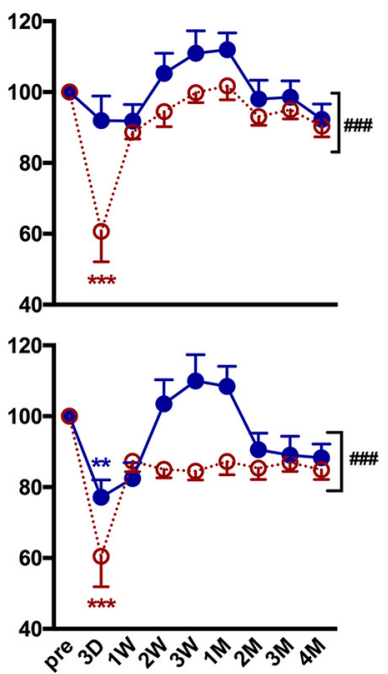

Swing Speed (\%)
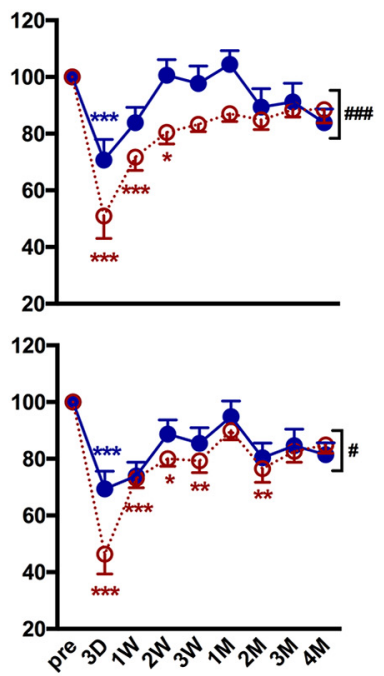

Figure 6. RLC improves motor and gait functions in chronic stroke. $\boldsymbol{A}$, RotaRod performance. $\boldsymbol{B}$, Regularity index to gauge coordination among limbs. $\boldsymbol{C}$, Gait analyses in affected forelimb and hindlimb. All behavior results are presented as a percentage of the preischemic baseline of each animal (\% pre). $n=19$ or 20/group. Two-way ANOVA: ${ }^{*} p<0.05$ versus pre (effect of stroke); ${ }^{* *} p<$ 0.01 versus pre (effect of stroke); ${ }^{* *} p<0.001$ versus pre (effect of stroke); $" p<0.05$ Sham versus RLC (effect of RLC); ${ }^{\# \#} p<0.01$ Sham versus RLC (effect of RLC); \#\#\# $p<0.001$ Sham versus RLC (effect of RLC).

Stroke severity determines the extent of RLC-induced monocyte shift

To understand the causative role of injury severity on RLCinduced proinflammatory monocyte shift, we tested whether stroke severity determines the extent of the RLC-induced monocyte shift. Separate cohorts of mice subjected to stroke received either Sham or RLC at $2 \mathrm{~h}$ after stroke, and both brain and serum were collected at $24 \mathrm{~h}$ according to the experimental timeline (Fig. 2A). Based on the infarct volume measured at $24 \mathrm{~h}$ after stroke, stroke/RLC mice were divided into moderate $\left(<25 \mathrm{~mm}^{3}\right)$ and severe $\left(\geq 30 \mathrm{~mm}^{3}\right)$ injury groups (Fig. $8 A$ ). There was no difference in monocyte subsets within splenocytes incubated with moderate and severely injured sera of Sham mice (Shammoderate vs Sham-severe, Ly-6C ${ }^{\text {low }} 49.84 \pm 3.97$ vs $42.48 \pm 2.89$, $p=0.1720 ;$ Ly- $6 C^{\text {high }} 27.16 \pm 2.54$ vs $\left.30.26 \pm 1.51, p=0.3245\right)$. Therefore, we combined results from Sham mice. Naive splenocytes incubated with RLC sera from moderate and severe injured mice showed a reduction of Ly- $6 \mathrm{C}^{\text {low }}$ monocytes and an increase in the Ly-6C $\mathrm{C}^{\text {high }}$ subset, with sera from severely injured mice generating the greatest shift (Fig. $8 B$ ). Notably, the ratio of Ly-6C $\mathrm{C}^{\text {high }}$ to Ly- $6 \mathrm{C}^{\text {low }}$ also showed dose-dependent monocyte shift (Fig. 8B). Together, these results showed that injury severity dictates the extent to which RLC induces a monocyte shift to promote functional recovery, indicating the importance of RLC-induced proinflammatory monocyte shift on stroke recovery.
CCR2-deficient splenocytes transfer abolishes RLC-mediated protection

To further investigate the importance of proinflammatory monocytes in RLC-induced benefits in stroke, CCR2 KO splenocytes were adoptively transferred to the asplenic C57BL/6 mice according to the previously described method (Kim et al., 2014). In nonstroked mice, RLC significantly shifted blood monocytes toward proinflammatory status in mice that received congenic splenocytes, but not in the mice that received CCR2 KO splenocytes (Fig. 9B). We further investigated the effect of CCR2 deficiency in stroke. In stroked mice that received CCR2-deficient splenocytes, RLC did not induce a monocyte shift to proinflammatory status in the blood or the brain (Fig. $9 C-E$ ). Animals that received CCR2-deficient splenocytes did not show RLCenhanced motor or gait recovery assessed up to 2 months, compared with Sham mice (Fig. 10). These results show that the CCR2-dependent proinflammatory monocyte shift is an underlying cellular mechanism for RLC-induced benefits after ischemic stroke.

\section{Discussion}

RLC has emerged as a noninvasive and clinically applicable strategy for cross-organ protection. Although several events are suggested to underlie conditioning-mediated protection (Zhou et al., 2011; Hoda et al., 2012, 2014), a clear mechanistic understanding by which RLC exerts protection has not been addressed. 

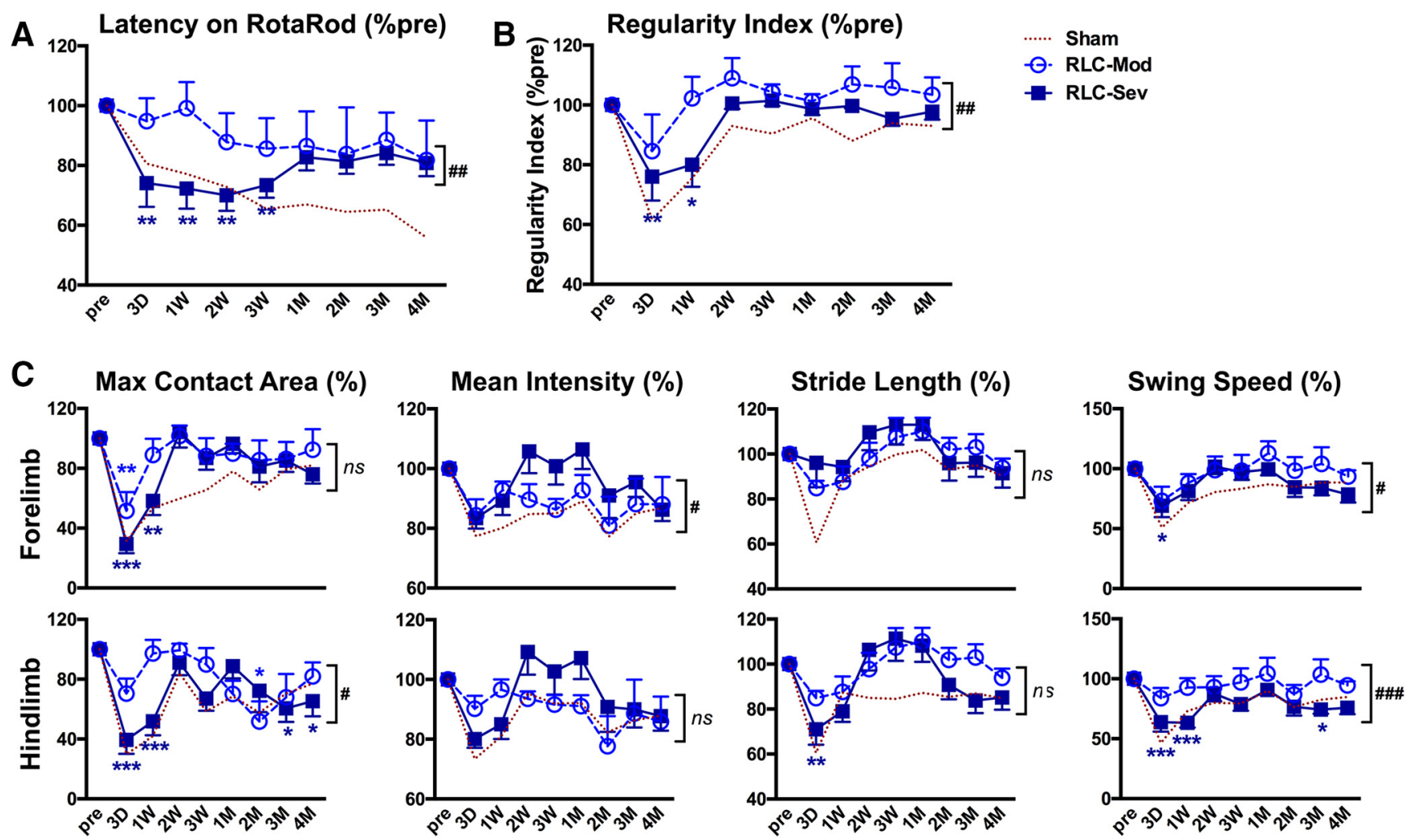

Figure 7. RLC-induced functional benefits occur independent of injury severity. RLC animals were divided into two groups, moderate and severe injury, based on the body weight reduction at $3 \mathrm{~d}$ after stroke ( $<15 \%$ loss for moderate injury and $\geq 15 \%$ loss for severe injury group). Dotted line, Behaviors of Sham mice for reference. $\boldsymbol{A}, \boldsymbol{B}, \operatorname{Rotarod}(\boldsymbol{A})$ and Regularity index ( $\boldsymbol{B})$ of Sham, RLC with a moderate injury, and RLC with severe injury mice. C, Gait analyses (maximum contact area on the glass plate, mean intensity exerted in the glass plate, stride length, and swing speed) of forelimb and hindlimb of the impaired side of the body (left side). All results are presented as a percentage of the preischemic baseline of each animal (\% pre). Two-way ANOVA: ${ }^{*} p<0.05$ versus pre (effect of stroke); ${ }^{* *} p<0.01$ versus pre (effect of stroke); ${ }^{* * *} p<0.001$ versus pre (effect of stroke); ${ }^{\#} p<0.05$ RLC-mod versus RLC-sev (effect of injury severity); ${ }^{\# \#} p<0.01$ RLC-mod versus RLC-sev (effect of injury severity); ${ }^{\# \#} p<0.001$ RLC-mod versus RLC-sev (effect of injury severity). Mod, Moderate injury; Sev, severe injury. ns, not significant.
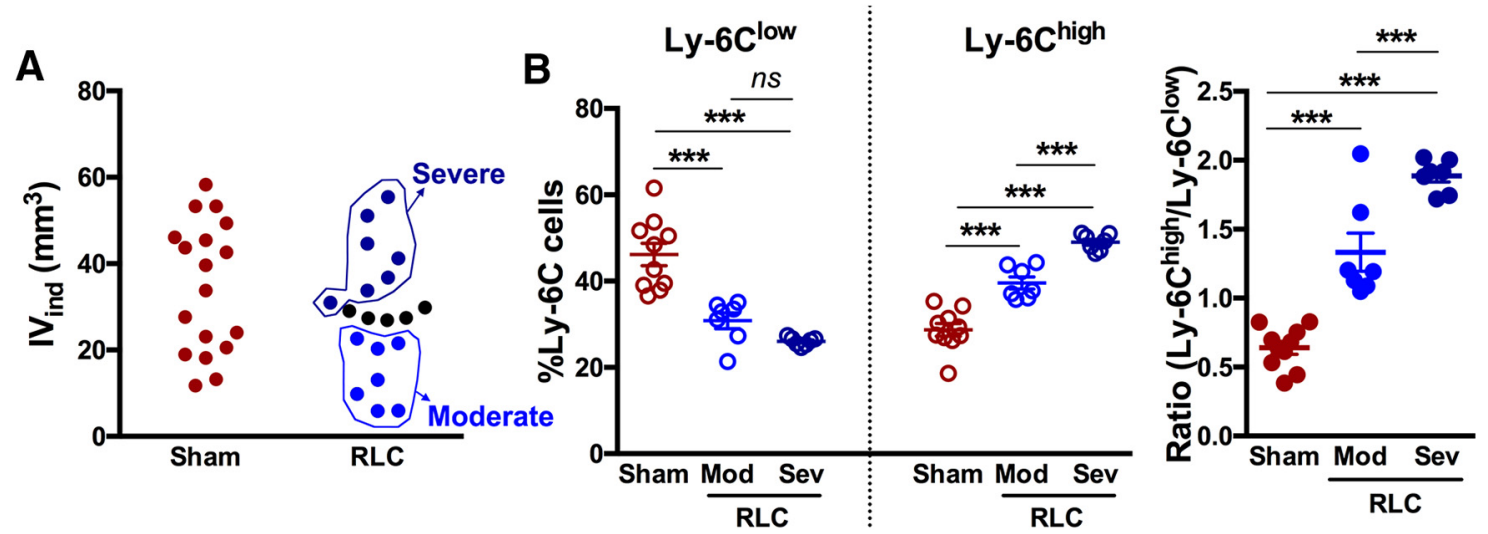

Figure 8. Stroke severity determines the extent of RLC-induced monocyte shift. $A$, Distribution of infarct volume in Sham and RLC mice. Animals with RLC were divided into two groups: moderate injury $\left(<25 \mathrm{~mm}^{3} \mathrm{IV}_{\text {ind }}\right)$ and severe injury $\left(\geq 30 \mathrm{~mm}^{3} \mathrm{IV}_{\text {ind }}\right) \cdot \boldsymbol{B}, \mathrm{Ly}-6 \mathrm{C}$ subset changes of naive CD11b ${ }^{+}$splenocytes incubated with poststroke serum from mice with Sham, RLC with moderate injury (Mod), or RLC with severe injury (Sev). ${ }^{* *} p<0.001$ (one-way ANOVA). IV ${ }_{\text {ind }}$ Indirect infarct volume; Mod, moderate injury; Sev, severe injury. ns, not significant.

In light of literature indicating the involvement of inflammatory mediators in conditioning-mediated protection, this study focused on RLC-induced peripheral monocyte inflammatory status changes, and addressed whether this change contributes to neuroprotection and functional recovery after stroke. Our major findings are that RLC produces an acute alteration in circulating monocytes toward a proinflammatory status, and this acute monocyte shift attenuates brain infarct and swelling and enhances long-term functional recovery. Notably, the extent of RLC-induced proinflammatory monocyte shift is injury severity- dependent, but behavioral improvement was observed regardless of the severity of injury. The absence of RLC-induced protection in mice that received CCR2-deficient splenocytes showed the importance of CCR2 ${ }^{+}$monocytes in RLC-enhanced functional recovery and defined a neuroimmune-based mechanism for RLC benefits in chronic ischemic stroke.

By applying RLC to naive mice, the study provided evidence of a proinflammatory monocyte shift in peripheral circulation. The monocyte subset shift was further confirmed in a closed culture system, where the monocyte trafficking to the injured brain is 
A
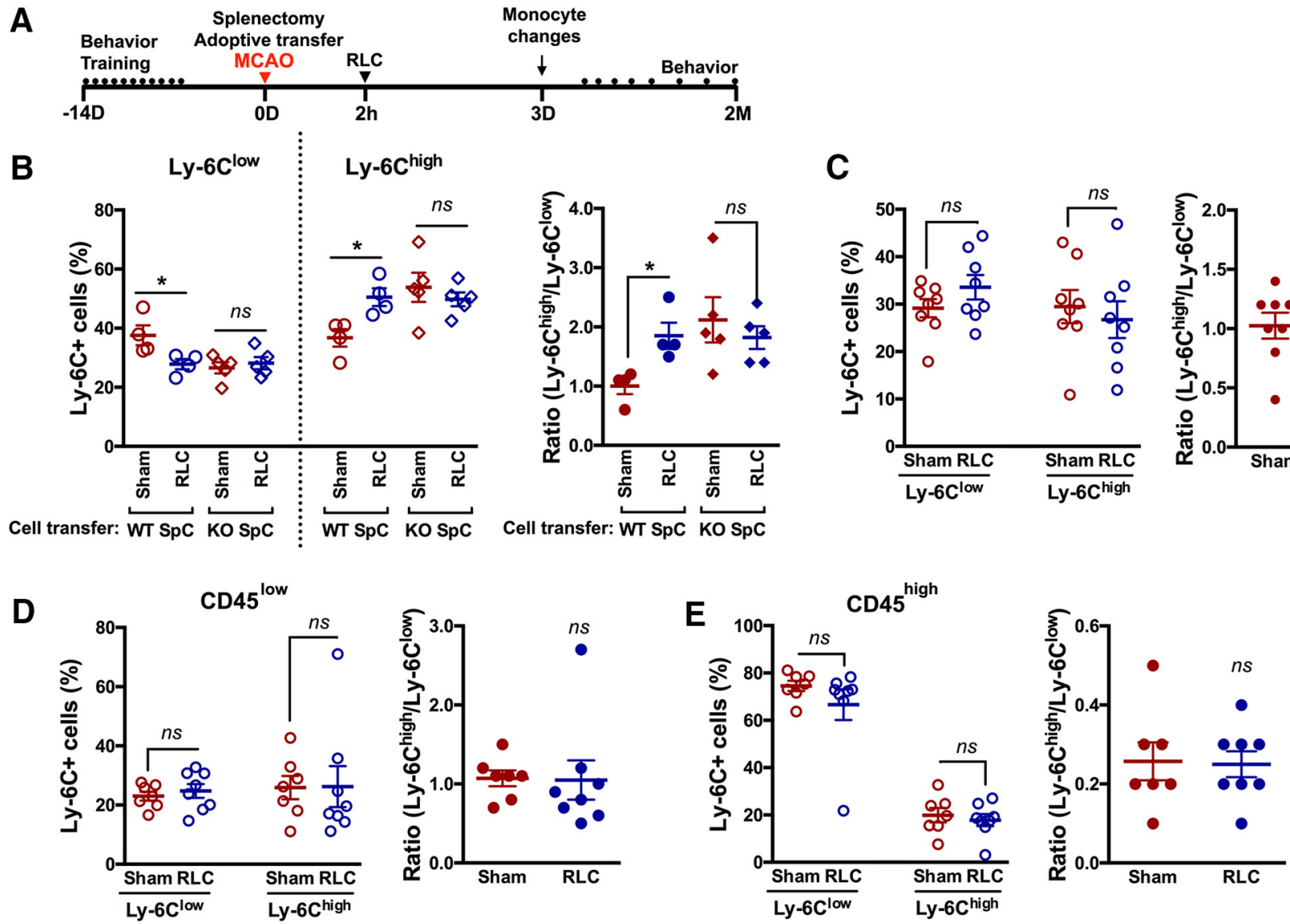

Figure 9. Adoptive transfer of CCR2-deficient splenocytes abolishes an RLC-induced proinflammatory monocyte shift. $\boldsymbol{A}$, Timeline of CCR2-deficient splenocyte adoptive transfer study. $\boldsymbol{B}$, Monocyte subsets and ratios of Ly- $6 C^{\text {high }} / \mathrm{Ly}-6 C^{\text {low }}$ in the blood of WT splenocytes transferred asplenic mice and CCR2 K0 splenocytes transferred asplenic mice. ${ }^{*} p<0.05$ (one-way ANOVA). $C$, Monocyte subset analysis in the blood of the CCR2 K0 splenocytes transferred to asplenic stroked mice after Sham or RLC. D, E, Percentage of Ly-6C subsets and ratios of Ly- $6 C^{\text {high }}$ over $L y-6 C^{\text {low }}$ subset in the $C^{2} 45^{\text {low }}(\boldsymbol{D})$ and $C D 45^{\text {high }}(\boldsymbol{E})$ population in the ipsilateral hemisphere. $t$ test. Contra, Contralateral hemisphere; Ipsi, ipsilateral hemisphere. ns, not significant.

absent. Previous adoptive transfer and detailed lineage tracing studies have shown that Ly-6C ${ }^{\text {high }}$ monocytes convert to Ly$6 \mathrm{C}^{\text {low }}$ monocytes in the blood and the bone marrow (Yona et al., 2013; Hilgendorf et al., 2014). Thus, our observation of increased Ly- $6 \mathrm{C}^{\text {high }} / \mathrm{CCR} 2{ }^{+}$monocytes raises the possibility that RLC may prevent the conversion of Ly-6 $\mathrm{C}^{\text {high }}$ monocytes to Ly-6C $\mathrm{C}^{\text {low }}$ monocytes. Alternatively, the observed reciprocal reduction of Ly- $6 C^{\text {low }}$ and increase of Ly- $6 C^{\text {high }}$ monocytes by treating RLC sera indicate a potential Ly- $6 \mathrm{C}^{\text {low }}$ to $\mathrm{Ly}-6 \mathrm{C}^{\text {high }}$ subset conversion in the blood. Our observation that this shift is maintained in an infiltrated CD $45^{\text {high }}$ population, but not in the resident CD $45^{\text {low }}$ population, within the stroked brain further supports that RLC shifts peripheral monocytes toward a proinflammatory state. While the conversion of Ly- $6 C^{\text {low }}$ to Ly- $6 C^{\text {high }}$ subset by RLC sera treatment indicates an involvement of humoral factor(s) in circulation, the identity of these upstream factors induced by RLC is presently unknown. Our initial assessment of MCP-1 protein levels in RLC sera was similar to sham sera (Sham: $502 \pm 214$ pg/ml, RLC: $515 \pm 390 \mathrm{pg} / \mathrm{ml}, n=7$ (group, not significant). We speculated that a combination of humoral factors acting in concert within a defined window is likely responsible for the monocyte shift in circulation. Future identification of a comprehensive set of upstream factors that cause the monocyte subset shift would potentially lead to drug development, a complementary pharmacological strategy for patients instead of the physical application of RLC.
Several mechanisms have been implicated in the RLCinduced benefits, including increased cerebral blood flow, increased nitrite in the blood, activated NMDAR, attenuated oxidative stress, and activated PI3K/Akt pathway (Zhou et al., 2011; Celso Constantino et al., 2014; Rassaf et al., 2014; Hess et al., 2016). With an emerging view of the critical role of inflammatory molecules in the stroke recovery (Chu et al., 2015; Wattananit et al., 2016), this study demonstrated that RLC improves acute stroke outcome and functional recovery. Despite excessive inflammation being tightly associated with injury development (Hughes et al., 2002; Dimitrijevic et al., 2006), our results indicate that sublethal stimulus by RLC-eliciting inflammation likely underlies the induction of ischemic tolerance. Inflammation is considered a necessary step for tissue remodeling and resolution (Zhao et al., 2006; Ekdahl et al., 2009). Our observation of a reduction in brain edema is accompanied by reduced MCP-1 and IL- $1 \beta$ gene expression, indicating that RLC may accelerates the resolution of inflammation.

Whether functional benefits in a chronic recovery of RLC animals are derived from the acute neuroprotection is less clear. The similar acute behavioral deficits between Sham and RLC mice indicate that RLC-induced functional recovery during the recovery phase is derived from events triggered by proinflammatory monocyte shift, rather than by acute neuroprotection itself. In several different types of stroke and myocardial infarction, the recruitment of proinflammatory monocytes into the injury site is 

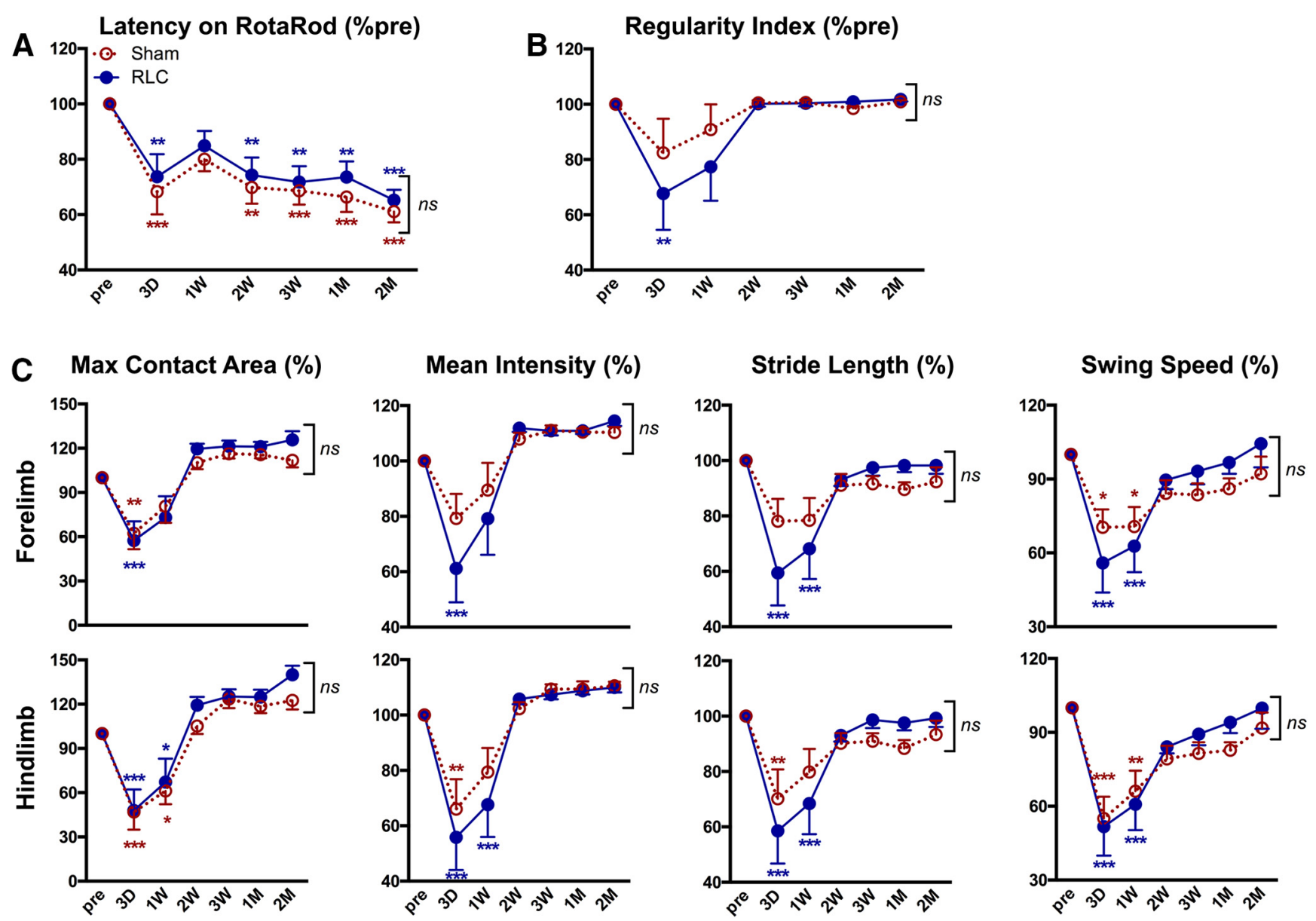

Figure 10. Adoptive transfer of CCR2-deficient splenocyte abolishes RLC-enhanced motor and gait recovery. $\boldsymbol{A}$, RotaRod performance. $\boldsymbol{B}$, Regularity index to gauge coordination among limbs. $\boldsymbol{C}$, Gait analyses in affected forelimb and hindlimb. All behavior results are presented as a percentage of the preischemic baseline of each animal (\% pre). $n=11-13 / \mathrm{group}$. Two-way ANOVA: ${ }^{*}<$ 0.05 versus pre (effect of stroke); ${ }^{* *} p<0.01$ versus pre (effect of stroke); ${ }^{* * *} p<0.001$ versus pre (effect of stroke). ns, not significant.

critical for late functional recovery (Hilgendorf et al., 2014; Chu et al., 2015; Wattananit et al., 2016). The lack of the circulating monocytes shifts at 4 months after stroke (Sham vs RLC, \%Ly$6 C^{\text {low }} 31.8 \pm 3.5$ vs $34.6 \pm 4.5, p=0.6320 ; \%$ Ly- $6 C^{\text {high }} 16.9 \pm 4.4$ vs $20.6 \pm 4.6, p=0.2942$ ) while there was persistent behavior improvement during the late recovery phase supports the potential importance of an early shift to CCR $2^{+}$subset by RLC for functional benefits in chronic stroke. The absence of monocyte shift and functional improvement in the asplenic mice that received CCR2-deficient splenocytes further supports the monocyte shift as a contributing cellular mechanism for RLC-induced protection.

Peripheral infection develops following ischemic stroke, which worsens neurological outcomes in both preclinical and clinical studies (Roth et al., 2001; Vermeij et al., 2009). While stroke severity, age, and dysphagia are known risk factors for poststroke infection, there is increasing evidence that poststroke immune depression triggers infections (Chamorro et al., 2007). While our study did not directly address the effect of RLC on peripheral infection, we speculate that increased proinflammatory monocytes by RLC may counteract stroke-induced immune depression and/or infection in the periphery. Since the infiltrated Ly- $6 \mathrm{C}^{\text {high }} / \mathrm{CCR} 2{ }^{+}$monocyte subset differentiates into reparative tissue macrophages (Chu et al., 2015), providing an adequate number of proinflammatory monocytes to the ischemic brain could facilitate a rapid and orchestrated immune response in the injured brain. This response, in turn, can lead to a resolution of inflammation and functional recovery.

Clinical trials are highly controlled and typically exclude severe stroke patients with either combined major comorbidities or adverse cardiovascular events (www.ClinicalTrials.gov, ID NCT03210051, NCT03231384, NCT03105141 etc.). This raises a potential safety issue and/or adverse effect on the application of RLC relevant injury severity. Based on a positive association between the extent of poststroke body weight loss and infarct volume, we used animals' body weight loss at $3 \mathrm{~d}$ to estimate acute injury severity and divided animals into two groups using a cluster analysis. While RLC mice with greater body weight loss (and therefore a bigger infarct) displayed greater overall acute deficits in motor/gait function, their functional recovery was similar to those with moderate body weight loss (smaller infarct). Interestingly, the greater RLC-induced proinflammatory shift with serum from animals with larger infarcts suggests that injury severity determines the extent of the monocyte shift and that the shift is causally linked to functional recovery in chronic stroke. Since no safety and feasibility concerns have been raised in human RLC trials (England et al., 2017; Zhao et al., 2017), our findings showing that RLC enhances behavioral recovery in both severe and moderately injured animals to a similar level suggests the potential for wide application of RLC in stroke patients.

Literature indicates the contributing role of proinflammatory monocytes in functional recovery as blocking CCR $2{ }^{+}$monocytes 
recruitment, delaying stroke recovery (Chu et al., 2015; Wattananit et al., 2016). Thus, we have further determined the role of CCR $2{ }^{+}$proinflammatory monocytes in RLC-induced monocyte shift and its effect on long-term stroke outcome. The RLCinduced beneficial effect was eliminated in mice that received CCR2-deficient monocytes, demonstrating a critical role of CCR $2{ }^{+}$monocytes for RLC-induced functional benefits. Consistent with our observation, a recent study addressing RLC effect in hemorrhagic stroke also showed that RLC-enhanced hematoma resolution was abolished in the absence of myeloid cells (Vaibhav et al., 2018), further supporting the role of circulating immune cells for the RLC-induced protective mechanism. One limitation of CCR2 KO adoptive transfer experiments is that we did not fully deplete CCR2 ${ }^{+}$monocytes from the host mice. WT splenectomized mice received CCR $2 \mathrm{KO}$ splenocytes, but there are sources of monocytes other than the spleen, including bone marrow and the blood. It is also possible that splenectomy and transfer of CCR2 KO monocytes into asplenic mice could have off-target effects, such as the alteration of the composition of immune cells (McBride et al., 1968). However, by applying Sham or RLC to identically prepared animals (splenectomy, CCR2 KO cell transfer, and MCAO) in the current study would provide the necessary role of CCR2 on RLC-induced benefits.

Limitations of the present findings include an undetermined relevance of other peripheral immune cells and their subsets. The absence of functional improvement in ischemic conditioned $\mathrm{T}$ cell-deficient nude rats, RLC-induced elevation of T cells, and NKT cells in the blood (Xie et al., 2013; Liu et al., 2016) indicates that other immune cells are involved in conditioning-induced benefits. Another limitation of this study is that the RLC therapeutic window was tested only at $2 \mathrm{~h}$ after stroke with animals that had normal metabolic status. Addressing wider therapeutic windows and incorporating comorbidities should be further explored to further assess the safety, feasibility, and translational efficacy of RLC. In addition, the upstream mediator(s) of the monocyte shift and the mechanism(s) by which acute RLCinduced monocyte shift exerts delayed functional improvement are unknown. An intriguing possibility is that the faster resolution of inflammation in the primary injury site (cortex and striatum) may attenuate secondary degeneration in the remote area. Indeed, secondary transneuronal degeneration in remote areas, such as substantia nigra and thalamus in stroke patients, is well documented (Ogawa et al., 1997; Ohe et al., 2013; Winter et al., 2015). The idea that RLC-induced functional improvement occurs via attenuation of secondary degeneration is intriguing and warrants future investigation.

In conclusion, the current study showed that RLC shifted the peripheral monocyte balance toward a proinflammatory status, and this shift led to an acute infarct volume and brain swelling reduction, as well as an enhancement of functional recovery in chronic stroke. RLC improved functional recovery across injury severity without any potential adverse events in subjects with severe injury. The observed RLC-induced changes in monocyte constituents were associated with improved stroke outcome. The absence of benefits in mice with CCR2-deficient cell transfer indicates not only the importance of proinflammatory monocytes in functional recovery in stroke but also RLC-induced functional benefits in stroke by shifting monocytes to a CCR $2{ }^{+}$proinflammatory subset. The functional benefits of RLC provide a platform for a neuroimmune-based strategy to modify the course of injury and enhance stroke recovery in patients.

\section{References}

Aslanyan S, Weir CJ, Diener HC, Kaste M, Lees KR (2004) Pneumonia and urinary tract infection after acute ischaemic stroke: a tertiary analysis of the GAIN international trial. Eur J Neurol 11:49-53.

Auffray C, Sieweke MH, Geissmann F (2009) Blood monocytes: development, heterogeneity, and relationship with dendritic cells. Annu Rev Immunol 27:669-692.

Bordet R, Deplanque D, Maboudou P, Puisieux F, Pu Q, Robin E, Martin A, Bastide M, Leys D, Lhermitte M, Dupuis B (2000) Increase in endogenous brain superoxide dismutase as a potential mechanism of lipopolysaccharide-induced brain ischemic tolerance. J Cereb Blood Flow Metab 20:1190-1196.

Celso Constantino L, Tasca CI, Boeck CR (2014) The role of NMDA receptors in the development of brain resistance through pre- and postconditioning. Aging Dis 5:430-441.

Chamorro A, Amaro S, Vargas M, Obach V, Cervera A, Torres F, Planas AM (2006) Interleukin 10, monocytes and increased risk of early infection in ischaemic stroke. J Neurol Neurosurg Psychiatry 77:1279-1281.

Chamorro A, Urra X, Planas AM (2007) Infection after acute ischemic stroke: a manifestation of brain-induced immunodepression. Stroke 38:1097-1103.

Chu HX, Broughton BR, Kim HA, Lee S, Drummond GR, Sobey CG (2015) Evidence that Ly6C(hi) monocytes are protective in acute ischemic stroke by promoting M2 macrophage polarization. Stroke 46:1929-1937.

Dimitrijevic OB, Stamatovic SM, Keep RF, Andjelkovic AV (2006) Effects of the chemokine CCL2 on blood-brain barrier permeability during ischemia-reperfusion injury. J Cereb Blood Flow Metab 26:797-810.

Dirnagl U, Becker K, Meisel A (2009) Preconditioning and tolerance against cerebral ischaemia: from experimental strategies to clinical use. Lancet Neurol 8:398-412.

Ekdahl CT, Kokaia Z, Lindvall O (2009) Brain inflammation and adult neurogenesis: the dual role of microglia. Neuroscience 158:1021-1029.

ElAli A, Jean LeBlanc N (2016) The role of monocytes in ischemic stroke pathobiology: new avenues to explore. Front Aging Neurosci 8:29.

England TJ, Hedstrom A, O’Sullivan S, Donnelly R, Barrett DA, Sarmad S, Sprigg N, Bath PM (2017) RECAST (Remote ischemic conditioning after stroke Trial): a pilot randomized placebo controlled phase II trial in acute ischemic stroke. Stroke 48:1412-1415.

Gliem M, Schwaninger M, Jander S (2016) Protective features of peripheral monocytes/macrophages in stroke. Biochim Biophys Acta 1862:329-338.

Gordon S (2007) Macrophage heterogeneity and tissue lipids. J Clin Invest 117:89-93.

Hess DC, Hoda MN, Khan MB (2016) Humoral mediators of remote ischemic conditioning: important role of eNOS/NO/Nitrite. Acta Neurochir Suppl 121:45-48.

Hilgendorf I, Gerhardt LM, Tan TC, Winter C, Holderried TA, Chousterman BG, Iwamoto Y, Liao R, Zirlik A, Scherer-Crosbie M, Hedrick CC, Libby P, Nahrendorf M, Weissleder R, Swirski FK (2014) Ly-6Chigh monocytes depend on Nr4al to balance both inflammatory and reparative phases in the infarcted myocardium. Circ Res 114:1611-1622.

Hoda MN, Siddiqui S, Herberg S, Periyasamy-Thandavan S, Bhatia K, Hafez SS, Johnson MH, Hill WD, Ergul A, Fagan SC, Hess DC (2012) Remote ischemic perconditioning is effective alone and in combination with intravenous tissue-type plasminogen activator in murine model of embolic stroke. Stroke 43:2794-2799.

Hoda MN, Bhatia K, Hafez SS, Johnson MH, Siddiqui S, Ergul A, Zaidi SK, Fagan SC, Hess DC (2014) Remote ischemic perconditioning is effective after embolic stroke in ovariectomized female mice. Transl Stroke Res 5:484-490.

Hughes PM, Allegrini PR, Rudin M, Perry VH, Mir AK, Wiessner C (2002) Monocyte chemoattractant protein-1 deficiency is protective in a murine stroke model. J Cereb Blood Flow Metab 22:308-317.

Jönsson AC, Lindgren I, Norrving B, Lindgren A (2008) Weight loss after stroke: a population-based study from the Lund Stroke Register. Stroke 39:918-923.

Kim E, Yang J, Beltran CD, Cho S (2014) Role of spleen-derived monocytes/ macrophages in acute ischemic brain injury. J Cereb Blood Flow Metab 34:1411-1419.

Kim E, Woo MS, Qin L, Ma T, Beltran CD, Bao Y, Bailey JA, Corbett D, Ratan RR, Lahiri DK, Cho S (2015) Daidzein augments cholesterol homeostasis via ApoE to promote functional recovery in chronic stroke. J Neurosci 35:15113-15126. 
Kono Y, Fukuda S, Hanatani A, Nakanishi K, Otsuka K, Taguchi H, Shimada $\mathrm{K}$ (2014) Remote ischemic conditioning improves coronary microcirculation in healthy subjects and patients with heart failure. Drug Des Dev Ther 8:1175-1181.

Liu ZJ, Chen C, Li XR, Ran YY, Xu T, Zhang Y, Geng XK, Zhang Y, Du HS, Leak RK, Ji XM, Hu XM (2016) Remote ischemic preconditioningmediated neuroprotection against stroke is associated with significant alterations in peripheral immune responses. CNS Neurosci Ther 22: 43-52.

McBride JA, Dacie JV, Shapley R (1968) The effect of splenectomy on the leucocyte count. Br J Haematol 14:225-231.

McColl BW, Rothwell NJ, Allan SM (2007) Systemic inflammatory stimulus potentiates the acute phase and CXC chemokine responses to experimental stroke and exacerbates brain damage via interleukin-1- and neutrophildependent mechanisms. J Neurosci 27:4403-4412.

McDonough A, Weinstein JR (2016) Neuroimmune response in ischemic preconditioning. Neurotherapeutics 13:748-761.

Ogawa T, Yoshida Y, Okudera T, Noguchi K, Kado H, Uemura K (1997) Secondary thalamic degeneration after cerebral infarction in the middle cerebral artery distribution: evaluation with MR imaging. Radiology 204:255-262.

Ohe Y, Uchino A, Horiuchi Y, Maruyama H, Deguchi I, Fukuoka T, Kato Y, Nagoya H, Dembo T, Tanahashi N (2013) Magnetic resonance imaging investigation of secondary degeneration of the mesencephalic substantia nigra after cerebral infarction. J Stroke Cerebrovasc Dis 22:58-65.

Ohtsuki T, Ruetzler CA, Tasaki K, Hallenbeck JM (1996) Interleukin-1 mediates induction of tolerance to global ischemia in gerbil hippocampal CA1 neurons. J Cereb Blood Flow Metab 16:1137-1142.

Oliveira RC, Brito MV, Ribeiro RF Jr, Oliveira LO, Monteiro AM, Brandão FM, Cavalcante LC, Gouveia EH, Henriques HY (2017) Influence of remote ischemic conditioning and tramadol hydrochloride on oxidative stress in kidney ischemia/reperfusion injury in rats. Acta Cir Bras 32: 229-235.

Qin L, Jing D, Parauda S, Carmel J, Ratan RR, Lee FS, Cho S (2014) An adaptive role for BDNF Val66Met polymorphism in motor recovery in chronic stroke. J Neurosci 34:2493-2502.

Rassaf T, Totzeck M, Hendgen-Cotta UB, Shiva S, Heusch G, Kelm M (2014) Circulating nitrite contributes to cardioprotection by remote ischemic preconditioning. Circ Res 114:1601-1610.

Roth EJ, Lovell L, Harvey RL, Heinemann AW, Semik P, Diaz S (2001) Incidence of and risk factors for medical complications during stroke rehabilitation. Stroke 32:523-529.

Starkopf J, Bugge E, Ytrehus K (1997) Preischemic bradykinin and ischaemic preconditioning in functional recovery of the globally ischaemic rat heart. Cardiovasc Res 33:63-70.

Sun J, Tong L, Luan Q, Deng J, Li Y, Li Z, Dong H, Xiong L (2012) Protective effect of delayed remote limb ischemic postconditioning: role of mitochondrial K(ATP) channels in a rat model of focal cerebral ischemic reperfusion injury. J Cereb Blood Flow Metab 32:851-859.

Urra X, Cervera A, Obach V, Climent N, Planas AM, Chamorro A (2009) Monocytes are major players in the prognosis and risk of infection after acute stroke. Stroke 40:1262-1268.
Vaibhav K, Braun M, Khan MB, Fatima S, Saad N, Shankar A, Khan ZT, Harris RB, Yang Q, Huo Y, Arbab AS, Giri S, Alleyne CH Jr, Vender JR, Hess DC, Baban B, Hoda MN, Dhandapani KM (2018) Remote ischemic post-conditioning promotes hematoma resolution via AMPKdependent immune regulation. J Exp Med 215:2636-2654.

Vermeij FH, Scholte op Reimer WJ, de Man P, van Oostenbrugge RJ, Franke CL, de Jong G, de Kort PL, Dippel DW, Netherlands Stroke Survey Investigators (2009) Stroke-associated infection is an independent risk factor for poor outcome after acute ischemic stroke: data from the Netherlands Stroke Survey. Cerebrovasc Dis 27:465-471.

Wang PF, Xiong XY, Chen J, Wang YC, Duan W, Yang QW (2015) Function and mechanism of toll-like receptors in cerebral ischemic tolerance: from preconditioning to treatment. J Neuroinflammation 12:80.

Wattananit S, Tornero D, Graubardt N, Memanishvili T, Monni E, Tatarishvili J, Miskinyte G, Ge R, Ahlenius H, Lindvall O, Schwartz M, Kokaia Z (2016) Monocyte-derived macrophages contribute to spontaneous longterm functional recovery after stroke in mice. J Neurosci 36:4182-4195.

Wei M, Xin P, Li S, Tao J, Li Y, Li J, Liu M, Li J, Zhu W, Redington AN (2011) Repeated remote ischemic postconditioning protects against adverse left ventricular remodeling and improves survival in a rat model of myocardial infarction. Circ Res 108:1220-1225.

Winter B, Brunecker P, Fiebach JB, Jungehulsing GJ, Kronenberg G, Endres M (2015) Striatal infarction elicits secondary extrafocal MRI changes in ipsilateral substantia nigra. PLoS One 10:e0136483.

Woo MS, Yang J, Beltran C, Cho S (2016) Cell-surface CD36 in monocyte/ macrophage contributes to phagocytosis during the resolution phase of ischemic stroke in mice. J Biol Chem 291:23654-23661.

Xie R, Wang P, Ji X, Zhao H (2013) Ischemic post-conditioning facilitates brain recovery after stroke by promoting Akt/mTOR activity in nude rats. J Neurochem 127:723-732.

Yang J, Kim E, Beltran C, Cho S (2019) Corticosterone-mediated body weight loss is an important catabolic process for poststroke immunity and survival. Stroke. Advance online publication. Retrieved July 26, 2019. doi: 10.1161/STROKEAHA.119.026053.

Yona S, Kim KW, Wolf Y, Mildner A, Varol D, Breker M, Strauss-Ayali D, Viukov S, Guilliams M, Misharin A, Hume DA, Perlman H, Malissen B, Zelzer E, Jung S (2013) Fate mapping reveals origins and dynamics of monocytes and tissue macrophages under homeostasis. Immunity 38 : 79-91.

Zhao BQ, Wang S, Kim HY, Storrie H, Rosen BR, Mooney DJ, Wang X, Lo EH (2006) Role of matrix metalloproteinases in delayed cortical responses after stroke. Nat Med 12:441-445.

Zhao W, Meng R, Ma C, Hou B, Jiao L, Zhu F, Wu W, Shi J, Duan Y, Zhang R, Zhang J, Sun Y, Zhang H, Ling F, Wang Y, Feng W, Ding Y, Ovbiagele B, Ji X (2017) Safety and efficacy of remote ischemic preconditioning in patients with severe carotid artery stenosis before carotid artery stenting: a proof-of-concept, randomized controlled Trial. Circulation 135:13251335 .

Zhou Y, Fathali N, Lekic T, Ostrowski RP, Chen C, Martin RD, Tang J, Zhang $\mathrm{JH}$ (2011) Remote limb ischemic postconditioning protects against neonatal hypoxic-ischemic brain injury in rat pups by the opioid receptor/Akt pathway. Stroke 42:439-444. 